\title{
Intervenção educativa para avaliação do conhecimento de enfermeiros intensivistas sobre lesão por pressão
}

\author{
Educational intervention to assess the knowledge of intensive care nurses about pressure \\ injury
}

Thiago Moura de Araújo ${ }^{1}$, Márcio Flávio Moura de Araújo ${ }^{1}$, Lívia Moreira Barros², Francisca Jane Gomes de Oliveira $^{3}$, Leonardo Alexandrino da Silva ${ }^{3}$, Joselany Áfio Caetano ${ }^{3}$

\begin{abstract}
Objetivo: comparar o conhecimento de enfermeiros intensivistas sobre lesões por pressão antes e após intervenção educativa. Métodos: estudo longitudinal, antes e depois, realizado com nove enfermeiros intensivistas alvos de intervenção educativa online sobre lesão por pressão. 0 conhecimento foi investigado por meio de instrumento com itens sobre prevenção, estadiamento e avaliação de lesões por pressão. Resultados: a média de acertos foi de 81,1\% e 84,6\%, na avaliação pré e pós-intervenção, respectivamente. Quanto à prevenção, houve média de acertos de 25,11 (78,5\%) antes da intervenção e 26,22 (81,9\%) após a realização do curso. Sobre o estadiamento, no pré-teste, obteve-se média de acertos de 4,33 questões (86,6\%), enquanto no pósteste a média foi de 4,66 (93,2\%). Na avaliação das lesões, os resultados foram iguais no pré e pós-intervenção. Conclusão: após realização do curso, verificou-se aumento no conhecimento dos enfermeiros sobre lesão por pressão, destacando-se os itens de estadiamento.
\end{abstract}

Descritores: Úlcera por Pressão; Educação a Distância; Capacitação em Serviço; Tecnologia da Informação; Enfermagem.

Objective: to compare the knowledge of intensive care nurses about pressure injuries before and after an educational intervention. Methods: longitudinal study before and after intervention, conducted with nine intensive care nurses target of an online educational intervention on pressure injuries. Knowledge was investigated through an instrument with items on prevention, staging and evaluation of pressure injuries. Results: the average proportion of correct answers was $81.1 \%$ and $84.6 \%$ in the pre- and post-intervention evaluation, respectively. Regarding prevention, there was an average of correct answers of $25.11(78.5 \%)$ before and $26.22(81.9 \%)$ after the intervention. As for staging, the average of correct answers was $4.33(86.6 \%)$ in the pre-test, and 4.66 (93.2\%) in the post-test. As for evaluation of injuries, the results were the same before and after the intervention. Conclusion: after the course, the knowledge of nurses about pressure injuries, particularly in items related to staging, increased.

Descriptors: Pressure Ulcer; Education, Distance; Inservice Training; Information Technology; Nursing.

\footnotetext{
${ }^{1}$ Universidade da Integração Internacional da Lusofonia Afro-Brasileira. Redenção, CE, Brasil.

${ }^{2}$ Universidade Estadual Vale do Acaraú. Sobral, CE, Brasil.

${ }^{3}$ Universidade Federal do Ceará. Fortaleza, CE, Brasil. 


\section{Introdução}

A lesão por pressão é concebida, no cenário da saúde pública, como um problema e um indicador da qualidade dos cuidados prestados. Além de causar dor e desconforto, essas lesões prolongam o período de internação e favorecem a morbimortalidade dos pacientes $^{(1-3)}$.

Nesse contexto, considera-se essencial que o enfermeiro tenha conhecimento e habilidades práticas e técnicas, além de atitudes positivas perante a ocorrência de eventos adversos ${ }^{(4)}$. A falta de conhecimentos e competências na prevenção de lesão por pressão contribui substancialmente para a sua ocorrência ou agravamento ${ }^{(5)}$.

Assim, baixo conhecimento e prática de enfermeiros contribuem para maior prevalência de lesões por pressão. Na prática clínica diária, a falta de conhecimento pode estar relacionada à baixa adesão às diretrizes, principalmente com relação à importância da mobilização e escolha da medida preventiva mais adequada $^{(2)}$.

Ademais, sendo a lesão por pressão um evento previsível e evitável que é prioridade na gestão do cuidado para a garantia da segurança do paciente, a implementação de programas educativos destinados a melhorar o conhecimento dos enfermeiros sobre essa temática é necessária e pode ter impacto na redução da sua elevada taxa de incidência e prevalência ${ }^{(6-7)}$.

Ao analisar a epidemiologia da lesão por pressão e a coexistência desse evento em unidades de terapia intensiva, torna-se pertinente o emprego de esforços relacionados à prevenção através de programas de educação permanente desenvolvidos a partir das tecnologias de informação e comunicação ${ }^{(1)}$.

A utilização desses tipos de tecnologias como instrumento estratégico nos programas de Educação Permanente em Saúde tem apresentado boa aceitação profissional. No entanto, essas ferramentas são relatadas em estudos, os quais não apresentam resultados de avaliação em curto e longo prazo ${ }^{(8)}$.
Acredita-se que a utilização das tecnologias de informação e comunicação, como método de relevância no processo de ensino-aprendizagem, pode ocorrer como um instrumento para a prática clínica do enfermeiro na avaliação e prevenção de lesões, capaz de contribuir para o empoderamento sobre a temática.

Diante do exposto, o objetivo deste estudo foi comparar o conhecimento de enfermeiros intensivistas sobre lesões por pressão antes e após intervenção educativa.

\section{Métodos}

Estudo longitudinal, conduzido antes e após intervenção educativa, realizado em um Hospital de Fortaleza, Ceará, Brasil. A intervenção foi aplicada com enfermeiros da unidade de terapia intensiva clínica por ser a unidade com maior prevalência de lesões por pressão na instituição, o que é associado à gravidade dos pacientes.

Os critérios de inclusão foram: ser enfermeiro assistencial em centro de terapia intensiva e ter acesso à internet para realização do curso. Os critérios de exclusão foram: curso de especialização na área de feridas, estar de licença médica/férias e não ter participado de pelo menos $80,0 \%$ no curso ou da avaliação ao final da intervenção educativa.

A população foi representada por 12 enfermeiros pertencentes à escala de trabalho, que concordaram em participar do estudo a partir de convite realizado pessoalmente e via correio eletrônico. Contudo, somente nove enfermeiros compuseram a amostra por conseguirem realizar todas as atividades propostas pelo curso e finalizá-lo. Os enfermeiros forneceram dados sociais, perfil profissional, conhecimento prévio sobre a temática e realização prévia de curso a distância. Os dados foram coletados em três fases, de janeiro a junho de 2015.

Na Fase I, entrevistaram-se os enfermeiros para identificar perfil profissional e conhecimento sobre lesão por pressão, por meio de questionário sociode- 
mográfico semiestruturado e instrumento para avaliação de conhecimento elaborado com base no Pressure Ulcer Knowledge Test validado no Brasil ${ }^{(9)}$. Ao final do curso, os enfermeiros avaliaram a tecnologia quanto à satisfação e atitudes após intervenção.

0 instrumento foi submetido à avaliação de conteúdo por três enfermeiras especialistas em feridas para desenvolvimento de escores de pontuação. Assim, cada afirmativa recebeu um escore que variou entre $1,0,1,5$ e 2,0. No questionário, as afirmativas 1 , $6,15,24,27,33,34,36,40-42$ receberam escore 2,0; as afirmativas $2,3,5,8,9,13,14,16,21,23,25,26,28$, 30,37 e 30, escore 1,5; e as afirmativas 4, 7, 10, 11, 12, $17,18,19,20,22,29,31,32,35$ e 39, escore 1,0. Criou-se peso para cada questão, levando em consideração o grau de dificuldade da resposta $(1,0 ; 1,5$ e 2,0 pontos) e a significância de cada item. Logo, identificou-se o nível de acertos das questões mais importantes. Ademais, os participantes que acertam mais questões com menor grau de complexidade apresentaram pontuação similar aos que acertaram número inferior.

Na fase II, os enfermeiros participaram da intervenção educativa e tiveram, inicialmente, 30 dias para concluir o curso. Entretanto, esse prazo foi prorrogado para 60 dias por falta de conclusão dos participantes devido à alta demanda de atividades e redução de tempo livre disponível para realização do curso, sendo enviadas notificações eletrônicas aos participantes cadastrados com 15 dias após realização do cadastro para enfatizar sobre a sua conclusão. Os participantes realizaram o cadastro na plataforma Moodle e, após, receberam e-mail com um código de acesso e instruções de como proceder.

O curso Úlcera por Pressão Online é uma tecnologia de informação e comunicação desenvolvida e validada pela Escola de Enfermagem da Universidade de São Paulo no Brasil para capacitar enfermeiros sobre prevenção e tratamento de Úlcera por Pressão, disponibilizado no Ambiente Virtual de Aprendizagem (AVA) Modular Object-Oriented Dynamic Learning Environment (Moodle), dividido em módulos que con- templam os seguintes tópicos: anatomia e fisiologia da pele; fisiopatologia da Úlcera por Pressão; análise de fatores de risco; escala de Braden e intervenções de enfermagem para sua prevenção; entre outros. Esse curso online exige do participante uma demanda em torno de 15 a $20 \mathrm{~h}$ para realização de todas as suas etapas $^{(10)}$.

Na fase III, após o término do período de intervenção, novamente aplicou-se o "Pressure Ulcer Knowledge Test" a fim de verificar a efetividade do curso "Úlcera por pressão online" no conhecimento dos enfermeiros intensivistas. Assim, a variável desfecho deste estudo foi o conhecimento adquirido pelos enfermeiros após a intervenção.

Os dados foram armazenados no programa Statistical Analysis System para cálculo de porcentagem, média, mediana e desvio padrão (DP \pm ). Na análise do pós-teste, utilizou-se o teste de McNemar, sendo considerados significativos aqueles com resultados de $p \leq 0,05$. 0 estudo foi aprovado pelo Comitê de Ética em Pesquisa da Universidade Federal do Ceará, sob parecer $\mathrm{n}$ - 1.075 .087 , em atendimento às normas da Resolução 466/12 sobre pesquisas que envolvem seres humanos.

\section{Resultados}

Todos os participantes foram do sexo feminino. A média de idade das enfermeiras foi de 36,11 anos, com variância entre 23 e 55 anos. A média do tempo de conclusão da graduação foi de 12,88 anos, sendo o máximo de 30 e o mínimo de dois anos. Nenhuma das participantes referiu histórico de realização de curso a distância relacionado à lesão por pressão/curativos.

Os itens acerca da prevenção totalizaram 32 itens, em que a média para os acertos foi de 25,11 $(78,5 \%)$ e 26,22 (81,9\%) no grupo pré e pós-intervenção, respectivamente. Na Tabela 1, são apresentadas as questões referentes ao conhecimento de medidas preventivas para lesão por pressão e a porcentagem de acertos antes e depois da intervenção educativa. 
Tabela 1 - Distribuição dos resultados referentes ao conhecimento sobre prevenção de lesão por pressão

\section{Prevenção de lesão por pressão}

\begin{tabular}{cccc} 
Pré-Teste & Pós-Teste & $\begin{array}{c}\text { Odds } \\
\text { Ratio }\end{array}$ & $\mathbf{p}^{*}$ \\
\hline $\mathbf{n}(\%)$ & $\mathbf{n}(\%)$ & \\
\hline
\end{tabular}

1. Amido de milho, cremes e curativos transparentes (tipo Tegaderm $®$ ou Opsite $®$ ) e curativos de hidrocoloides não protegem contra os efeitos da fricção da pele $(\mathrm{F})^{\dagger}$

5. Rodas d'água ou almofadas tipo argola auxiliam na prevenção de úlcera por pressão (F)

8. Todos os indivíduos com risco para úlcera por pressão devem ter uma inspeção sistemática da pele pelo menos uma vez por semana (F)

9. A cabeceira da cama deve ser mantida em um baixo grau de elevação (de preferência, não maior que um ângulo de 30 graus) consistente com as condições médicas (V) *

10. Na posição lateral, a pessoa deve ficar em ângulo de 30 graus com a cama (V)

19. Todos os indivíduos devem ser avaliados na admissão no hospital quanto ao risco para desenvolver úlcera por pressão (V)

20. Uma ingestão dietética adequada de proteínas e calorias deve ser mantida durante a doença (V)

21. Protetores de calcâneos como luvas d'água aliviam a pressão nos calcâneos (F)

22. A pele deve permanecer limpa e seca (V)

23. Medidas de prevenção não necessitam ser usadas para prevenir novas lesões quando o paciente já possui úlcera por pressão $(\mathrm{F})$

24. A mobilização e a transferência de pacientes totalmente dependentes devem ser feitas por duas ou mais pessoas $(\mathrm{V})$

25. Todo paciente admitido na Unidade de Terapia Intensiva deve ser submetido à avaliação do risco para o desenvolvimento de úlcera por pressão (V)

26. Uma boa maneira de diminuir a pressão nos calcâneos é elevá-los do leito (V)

27. Todo o cuidado administrado para prevenir ou tratar úlceras por pressão não necessita ser documentado $(\mathrm{F})$

28. Cisalhamento é a força que ocorre quando a pele adere a uma superfície e o corpo desliza (V)

29. A fricção pode ocorrer ao movimentar a pessoa para cima na cama (V)

30. Para as pessoas que têm incontinência, a limpeza da pele deve ocorrer no momento em que se sujar e nos intervalos de rotina (V)

31. Programas educacionais podem reduzir a incidência de úlceras por pressão (V)

32. Pacientes hospitalizados precisam ser avaliados quanto ao risco para úlcera por pressão uma única vez $(\mathrm{F})$

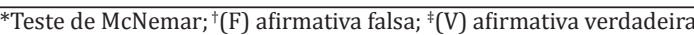

Entre as 32 questões de prevenção, identificaram-se $14(43,7 \%)$ respondidas de forma correta por todos os participantes, antes e após a intervenção. Três itens do instrumento apresentaram diminuição nos acertos quanto à avaliação após a intervenção. Ainda assim, o nível de acerto foi mantido acima de $60,0 \%$, sendo sete $(78,0 \%)$ acertos no grupo pré-teste e seis $(67,0 \%)$ no grupo pós-teste (Tabela 1$)$.

$2(22,0) \quad 1(11,0) \quad 4,00 \quad 0,113$

$6(67,0) \quad 6(67,0) \quad 0,5 \quad 0,505$

$8(89,0) \quad 7(78,0) \quad 7,00 \quad 0,077$

$7(78,0) \quad 6(67,0) \quad 3,00 \quad 0,288$

$4(44,0) \quad 8(89,0) \quad 1.6 \quad 0,579$

$9(100,0) \quad 9(100,0) \quad 1,00 \quad 1,000$

$9(100,0) \quad 9(100,0) \quad 1,00 \quad 1,000$

$9(100,0) \quad 9(100,0) \quad 1,00 \quad 1,000$

$9(100,0) \quad 9(100,0) \quad 1,00 \quad 1,000$

$9(100,0) \quad 9(100,0) \quad 1,00 \quad 1,000$

$9(100,0) \quad 9(100,0) \quad 1,00 \quad 1,000$

$9(100,0) \quad 9(100,0) \quad 1,00 \quad 1,000$

$9(100,0) \quad 9(100,0) \quad 1,00 \quad 1,000$

$9(100,0) \quad 9(100,0) \quad 1,00 \quad 1,000$

$9(100,0) \quad 9(100,0) \quad 1,00 \quad 1,000$

$9(100,0) \quad 9(100,0) \quad 1,00 \quad 1,000$

$9(100,0) \quad 9(100,0) \quad 1,00 \quad 1,000$

$9(100,0) \quad 9(100,0) \quad 1,00 \quad 1,000$
$9(100,0) \quad 9(100,0) \quad 1,00 \quad 1,000$ 
respostas certas foi acerca da manutenção da angulação de 30 graus da cama para pacientes na posição lateral (de 44,0\% para 89,0\%). 0 item escala de horários para mudança de decúbito, bem como o período para reposicionamento do paciente em cadeira de
A alteração do conhecimento sobre as úlceras por pressão de estágio II que podem ser extremamente dolorosas devido à posição das terminações nervosas foi ampliada, com significância estatística, conforme observado.

Tabela 2 - Distribuição dos resultados referentes ao estadiamento da lesão por pressão

\begin{tabular}{|c|c|c|c|c|}
\hline \multirow{2}{*}{ Estadiamento da lesão por pressão } & \multirow{2}{*}{$\frac{\text { Pré-teste }}{n(\%)}$} & Pós-teste & \multirow{2}{*}{$\begin{array}{l}\text { Odds } \\
\text { Ratio }\end{array}$} & \multirow{2}{*}{$\mathbf{p}^{*}$} \\
\hline & & $n(\%)$ & & \\
\hline 33. 0 estágio I da úlcera por pressão é definido como um eritema que não embranquece $(\mathrm{V})^{\dagger}$ & $7(78,0)$ & $8(89,0)$ & 4,00 & 0,113 \\
\hline 34. Uma úlcera por pressão em estágio III é uma perda parcial de pele envolvendo a epiderme (V) & $6(67,0)$ & $8(89,0)$ & 2,66 & 0,227 \\
\hline $\begin{array}{l}\text { 35. Úlceras por pressão no estágio IV apresentam uma perda de pele total com intensa destruição } \\
\text { e necrose tissular ou danos aos músculos, ossos ou estruturas de suporte (V) }\end{array}$ & $9(100,0)$ & $9(100,0)$ & 1,00 & 1,000 \\
\hline 36. Uma bolha no calcâneo não deve ser motivo de preocupação $(F)^{\ddagger}$ & $9(100,0)$ & $9(100,0)$ & 1,00 & 1,000 \\
\hline $\begin{array}{l}\text { 37. As úlceras por pressão de estágio II podem ser extremamente doloridas pela posição das } \\
\text { terminações nervosas (V) }\end{array}$ & $8(89,0)$ & $8(89,0)$ & 8,00 & 0,045 \\
\hline
\end{tabular}

Tabela 3 - Distribuição dos resultados referentes à avaliação da lesão por pressão

\begin{tabular}{|c|c|c|c|c|}
\hline \multirow{2}{*}{ Avaliação da lesão por pressão } & Pré-teste & Pós-teste & \multirow{2}{*}{$\begin{array}{l}\text { Odds } \\
\text { Ratio }\end{array}$} & \multirow{2}{*}{$\mathbf{p}^{*}$} \\
\hline & $\mathbf{n}(\%)$ & n(\%) & & \\
\hline 38. As úlceras por pressão são feridas estéreis $(\mathrm{F})^{\dagger}$ & $7(78,0)$ & $7(78,0)$ & 3,50 & 0,182 \\
\hline 39. Uma cicatriz de úlcera por pressão poderá lesar mais rapidamente que a pele íntegra $(\mathrm{V})^{\ddagger}$ & $9(100,0)$ & $9(100,0)$ & 1,00 & 1,000 \\
\hline 40. A úlcera por pressão pode ocorrer no paciente após 24 horas de sua internação (V) & $6(67,0)$ & $6(67,0)$ & 2,00 & 0,505 \\
\hline 41. A região sacral e o calcâneo são as mais acometidas por úlceras por pressão (F) & $8(89,0)$ & $8(89,0)$ & 8,00 & 0,045 \\
\hline $\begin{array}{l}\text { 42. Pode-se indicar a utilização de hidrogel somente em úlceras por pressão cavitárias e com } \\
\text { tecido de granulação presente (F) }\end{array}$ & $4(44,0)$ & $4(44,0)$ & 0,80 & 1,000 \\
\hline
\end{tabular}

rodas, foi corretamente respondido por três $(33,3 \%)$ participantes antes e depois a intervenção educativa (Tabela 1). Quanto ao estadiamento, o grupo pré-teste apresentou média de acertos de 4,33 questões $(86,6 \%)$ contra $4,66(93,2 \%)$ do grupo pós-teste. 0 item que apresentou maior diferença de percentual de acertos antes e depois do curso trata-se daquele referente à caracterização da lesão em estágio III, com seis $(67,0 \%)$ e oito $(89,0 \%)$ acertos, no grupo pré e no pós-teste, respectivamente; seguido pelo item referente à dificuldade de diferenciação da lesão por pressão em estágio I, com sete $(78,0 \%)$ e $8(89,0 \%)$ acertos. Porém, 100,0\% das enfermeiras acertaram a descrição em estágio III e IV, antes e depois da intervenção educativa (Tabela 2).
No último grupo de questões relacionadas à lesão por pressão estão cinco itens referentes à avaliação. As enfermeiras apresentaram resultado igual na fase pré e pós-intervenção educativa para esses itens, com uma média de acertos de 3,77 (75,4\%). 0 item sobre indicação do hidrogel em lesões cavitárias e com tecido de granulação apresenta-se como uma afirmação errada, contudo menos da metade das enfermeiras acertaram esse item. Somente o item a respeito da maior chance de desenvolver esse tipo de lesão em regiões com cicatriz de lesões anteriores, em relação à pele íntegra, apresentou $100,0 \%$ de acertos em ambas as avaliações (Tabela 3).

A porcentagem de acertos das enfermeiras na primeira avaliação foi superior a 69,0\%, atingindo 
até $88,0 \%$. Na segunda avaliação, depois da intervenção educativa, as enfermeiras apresentaram mais de $73,0 \%$ de acertos, inclusive uma enfermeira com mais de $95,0 \%$.

A porcentagem de acertos entre as nove participantes teve uma média de $81,1 \%$ e $84,6 \%$, na avaliação pré e pós-intervenção, respectivamente. Os itens avaliados referentes à prevenção apresentaram $80,9 \%$ e $84,8 \%$ de acertos; já os itens de estadiamento obtiveram $86,8 \%$ e $93,4 \%$ de acertos, nos grupos pré e pós-intervenção, respectivamente. Os itens referentes à avaliação de lesão por pressão não apresentaram mudança após a intervenção, com uma média de pontuação de $75,6 \%$.

Após a intervenção educativa, o conhecimento referente à identificação da região mais acometida por úlceras por pressão, embora a quantidade de enfermeiras que acertaram tenha sido igual, oito $(89,0 \%)$, houve associação significativa.

A média de pontuação das enfermeiras aumentou mais de 5 pontos, comparativamente, entre a primeira e a segunda avaliação. A média de questões erradas na primeira avaliação ( $M D=5,88 ; \mathrm{DP} \pm 2,57)$ foi superior à da segunda avaliação $(\mathrm{MD}=5,0 ; \mathrm{DP} \pm 2,54)$. A média de questões marcadas como "não sabe" foi de 2,44 (DP $\pm 1,81)$ e 1,22 (DP $\pm 2,16)$ na avaliação pré e pós-intervenção, respectivamente.

\section{Discussão}

Destacam-se como limitações do estudo o quantitativo de enfermeiros que compuseram a amostra final e o recorte pontual na avaliação das variáveis, o que pode representar obstáculo na generalização dos resultados em outros contextos.

Infere-se que a estratégia de treinamento e educação continuada sobre lesão por pressão por meio de tecnologia de informação e comunicação apresenta-se como um modelo promissor, visto que aumenta as opções de meios de empoderamento dos enfermeiros e, consequentemente, garante mais qualidade e efetividade.
A realização de treinamentos e capacitações para aquisição de conhecimentos por meio de educação continuada é certamente uma das formas mais seguras de beneficiar os funcionários de uma empresa e gerar produtividade e/ou aumento da qualidade da assistência. Estudo realizado na Jordânia para avaliar a eficácia do e-learning como método de ensino para melhorar as habilidades dos enfermeiros na classificação de lesão por pressão verificou que, no pós-teste, o grupo experimental apresentou média mais elevada na pontuação em comparação com o controle ${ }^{(7)}$.

Em ensaio clínico controlado e randomizado realizado na Espanha para avaliar a eficácia de um programa e-learning no ensino sobre lesões por pressão em comparação com o método tradicional, os resultados demonstraram que os alunos de enfermagem do grupo e-learning apresentaram melhores médias no pós-teste quando comparados com os alunos do grupo de ensino tradicional ${ }^{(11)}$.

Observa-se no presente estudo um bom conhecimento dos profissionais sobre a prevenção de lesão por pressão antes e depois da intervenção educativa. Prevenir essas lesões é uma das principais atividades da equipe de enfermagem. Uma decisão baseada em evidências é fundamentada em pesquisas válidas e relevantes, nas preferências do paciente, nos recursos disponíveis e no julgamento e experiência do enfermeiro $^{(3)}$.

Para prevenir essas lesões, o enfermeiro deve possuir conhecimento sobre os fatores de risco, sendo isso um pré-requisito para o cuidado de alta qualidade, uma vez que a falta de conhecimento pode resultar em equívocos na prestação dos $\operatorname{cuidados}^{(7)}$. Nesse contexto, o conhecimento adequado sobre prevenção é fundamental, o qual irá nortear a identificação do enfermeiro quanto aos pacientes que estão ou não em risco e quais medidas preventivas devem ser aplica$\mathrm{das}^{(5)}$.

Ao analisar as perguntas do questionário, observa-se que os enfermeiros desempenham um papel importante na prevenção e tratamento de lesão por pressão por estarem diretamente envolvidos na im- 
plementação de cuidados como avaliação do risco e reposicionamento do paciente ${ }^{(12)}$. 0 uso de superfícies de suporte em camas e cadeiras para facilitar a redistribuição da pressão e o reposicionamento são importantes medidas preventivas, principalmente para os pacientes com baixa mobilidade, devendo ser utilizadas em conjunto ${ }^{(13)}$.

Durante o ensino teórico e as aulas práticas, a classificação da lesão por pressão deve ser enfatizada para melhorar a precisão do enfermeiro na prática assistencial e nas pesquisas sobre a temática ${ }^{(4)}$. A tomada de decisão sobre as intervenções adequadas para cada paciente pode ser dificultada devido à incerteza do profissional em classificar corretamente a lesão e, consequentemente, escolher as medidas preventivas ou o tratamento $0^{(9)}$. As afirmativas relacionadas à avaliação da lesão por pressão não proporcionaram mudança no conhecimento dos enfermeiros, apresentando uma pontuação baixa sobre indicação de produto para tratamento e outro sobre o tempo de ocorrência após internação.

Quando os enfermeiros não apresentam conhecimento claro sobre as causas da lesão por pressão e seus fatores de risco, os pacientes não podem esperar receber cuidados preventivos baseados em evidências. A identificação correta dos seus fatores de risco nos pacientes e a implementação de intervenções de enfermagem adequadas irão impactar na sua incidência na Unidade de Terapia Intensiva, influenciando diretamente na qualidade do cuidado e na melhoria da segurança do paciente ${ }^{(3,6)}$.

Assim, quando o paciente apresenta fatores de risco para o desenvolvimento de lesão por pressão, a meta principal da equipe de saúde é elaborar um plano de cuidados com o paciente para prevenir a ocorrência da lesão. A escolha dos cuidados deve ser baseada nas necessidades de cada paciente e na avaliação de risco em que se recomenda o uso de instrumentos padronizados e validados ${ }^{(12)}$.

A avaliação do questionário utilizado aponta que tanto no pré como no pós-teste os participantes deste estudo apresentaram um bom conhecimento sobre a temática. Na Arábia Saudita, estudo que verificou o conhecimento e a atitude de 105 profissionais de saúde sobre prevenção de lesão por pressão em um hospital de reabilitação aguda com o mesmo instrumento teve como pontuação média de acertos 34,1 (71,5\%). Classificando-se a média da porcentagem de cada categoria profissional, os médicos tiveram 79,3\% de acertos, os enfermeiros 75,0\%, 71,7\% para os terapeutas ocupacionais e $58,5 \%$ para os fisioterapeutas, demonstrando que os enfermeiros possuem conhecimento adequado sobre a prevenção da lesão em questão ${ }^{(5)}$.

Estudo similar foi realizado no Irã, o qual analisou o conhecimento sobre a prevenção, classificação e gestão de lesão por pressão de 159 enfermeiros do setor de trauma de um hospital de emergência e obteve uma média de porcentagem de acertos de 64,6\%, inferior ao do presente estudo. Observou-se que os enfermeiros tinham maior conhecimento sobre as características da ferida (avaliação) e conhecimento deficiente sobre as causas e fatores de risco para o seu surgimento ${ }^{(14)}$.

O baixo nível de conhecimento dos enfermeiros sobre prevenção e tratamento de lesão por pressão está associado à formação dos alunos na graduação, o que põe em risco os pacientes que estão sendo assistidos por esses profissionais ao considerar que o cuidado da pele está diretamente relacionado à equipe de enfermagem. A avaliação de competências específicas para a prevenção de lesões por pressão, em particular no momento da graduação, é parte da garantia do ensino de enfermagem de qualidade ${ }^{(1,15)}$.

\section{Conclusão}

Verificou-se, após a realização do curso online sobre lesão por pressão, aumento no conhecimento dos enfermeiros e a pontuação que apresentou maior significância é referente ao estadiamento. 


\section{Colaborações}

Araújo TM e Araújo MFM contribuíram na concepção do projeto. Oliveira FJG e Silva LA colaboraram na redação e revisão crítica do conteúdo intelectual. Barros LM e Caetano JA auxiliaram na revisão crítica do conteúdo intelectual e aprovação final da versão a ser publicada.

\section{Referências}

1. Vasconcelos JMB, Caliri MHL. Nursing actions before and after a protocol for preventing pressure injury in intensive care. Esc Anna Nery. 2017; 21(1):e20170001. doi: http://dx.doi. org/10.5935/1414-8145.20170001

2. Nuru N, Zewdu F, Amsalu S, Mehretie Y. Knowledge and practice of nurses towards prevention of pressure ulcer and associated factors in Gondar University Hospital, Northwest Ethiopia. BMC Nurs. 2015; 14(34):1-8. doi: http://dx.doi. org/10.1186/s12912-015-0076-8

3. Gunningberg L, Mårtensson G, Mamhidir AG, Florin J, Muntlin Å̊, Bååth C. Pressure ulcer knowledge of registered nurses, assistant nurses and student nurses: a descriptive, comparative multicentre study in Sweden. Int Wound J. 2015; 12(4):462-8. doi: dx.doi.org/10.1111/iwj.12138

4. Bredesen IM, Bjøro K, Gunningberg L, Hofoss D. Effect of e-learning program on risk assessment and pressure ulcer classification-A randomized study. Nurse Educ Today. 2016; 40:191-7. doi: http://dx.doi.org/10.1016/j.nedt.2016.03.008

5. Kaddourah B, Abu-Shaheen AK, Al-Tannir M. Knowledge and attitudes of health professionals towards pressure ulcers at a rehabilitation hospital: a cross-sectional study. BMC Nurs. 2016; 15:17. doi: http://dx.doi.org/10.1186/s12912016-0138-6

6. Tayyib N, Coyer F, Lewis PA. A two-arm cluster randomized control trial to determine the effectiveness of a pressure ulcer prevention bundle for critically ill patients. J Nurs Scholarsh. 2015; 47(3):237-47. doi: http://dx.doi.org/ dx.doi.org/10.1111/jnu.12136

7. Tubaishat A. The Effectiveness of an e-Learning Program to Improve Pressure Ulcer Classification by Nurses. Int J Humanit Soc Sci [Internet]. 2014 [cited May 13, 2019]; 4(10):293-300. Available from: http://www.ijhssnet.com/journals/Vol_4_ No_10_1_August_2014/32.pdf

8. Gonçalves MBB, Rabeh SAN, Terçariol CAS. The contribution of distance learning to the knowledge of nursing lecturers regarding assessment of chronic wounds. Rev Latino-Am Enfermagem. 2015; 23(1):122-9. doi: http:// dx.doi.org/10.1590/0104-1169.3606.2533

9. Rabeh SAN, Palfreyman S, Souza CBL, Bernardes RM, Caliri MHL. Cultural adaptation of the PieperZulkowski pressure ulcer knowledge test for use in Brazil. Rev Bras Enferm. 2018; 71(4):1977-84. doi: dx.doi.org/10.1590/0034-7167-2017-0029

10. Costa JB, Peres HHC, Rogenski NMB, Baptista $\mathrm{CMC}$. An educational proposal to teach a pressure ulcer management course online to students and nursing professionals. Acta Paul Enferm. 2009; 22(5):607-11. doi: http://dx.doi.org/10.1590/ S0103-21002009000500002

11. Bredesen IM, Bjøro K, Gunningberg L, Hofoss D. The prevalence, prevention and multilevel variance of pressure ulcers in Norwegian hospitals: a cross-sectional study. Int J Nurs Stud. 2015; 52(1):149-56. doi: http://dx.doi.org/10.1016/j. ijnurstu.2014.07.005

12. Samuriwo R, Dowding D. Nurses' pressure ulcer related judgements and decisions in clinical practice: a systematic review. Int J Nurs Stud. 2014; 51(12):1667-85. doi: http://dx.doi. org/10.1016/j.ijnurstu.2014.04.009

13. Morente L, Morales-Asencio JM, Veredas FJ. Effectiveness of an e-learning tool for education on pressure ulcer evaluation. J Clin Nurs. 2014; 23(13-14):2043-52. doi: http://dx.doi. org/10.1111/jocn.12450

14. Rafiei H, Abdar ME, Iranmanesh S, Lalegani H, Safdari A, Dehkordi AH. Knowledge about pressure ulcer prevention, classification and management: a survey of registered nurses working with trauma patients in the emergency department. Int J Orthop Trauma Nurs. 2014; 18(3):135-42. doi: http://dx.doi.org/10.1016/j.ijotn.2014.03.004

15. Caldini LN, Araújo TM, Frota NM, Barros LM, Silva LA, Caetano JÁ. Evaluation of educational technology on pressure injury based on assistance quality indicators. Rev Rene. 2018; 19(1):e32695. doi:http://dx.doi.org/10.15253/21756783.20181932695 